\title{
ПРЕДЛОЖЕНИЯ ПО СОЗДАНИЮ ВЫСОТНОГО ГЕОДЕЗИЧЕСКОГО ОБОСНОВАНИЯ ДЛЯ ДЕФОРМАЦИОННОГО МОНИТОРИНГА ОБЪЕКТОВ КАПИТАЛЬНОГО СТРОИТЕЛЬСТВА
}

\section{Евгений Ильич Аврунев}

Сибирский государственный университет геосистем и технологий, 630108, Россия, г. Новосибирск, ул. Плахотного, 10, кандидат технических наук, директор Института кадастра и природопользования, тел. (383)344-31-73, e-mail: kadastr204@yandex.ru

\section{Павел Сергеевич Батин}

ООО «Геосити», 630054, Россия, г. Новосибирск, ул. Титова 29/1, директор, тел. (903)999-38-26, e-mail: batin866@mail.ru

\section{Николай Олегович Гревцев}

Сибирский государственный университет геосистем и технологий, 630108, Россия г. Новосибирск, ул. Плахотного, 10, обучающийся, тел. (960)797-32-33, e-mail: kadastr204@yandex.ru

В статье определена актуальность выполнения тригонометрического нивелирования при геодезическом мониторинге. На конкретном производственном объекте выполнено сравнение точности тригонометрического нивелирования, выполненного с использованием двух электронных тахеометров, с высокоточным геометрическим нивелированием, которое построено по программе первого класса. На основании сравнения вычисленных превышений с эталонными значениями, полученными из высокоточного геометрического нивелирования, определена реальная точность тригонометрического нивелирования и эффективность предложенного математического алгоритма для определения допусков при использовании этого наиболее технологического способа определения пространственного положения объектов недвижимости. Предложенный математический алгоритм целесообразно использовать при проведении геодезического мониторинга.

Ключевые слова: объект капитального строительства, деформационный мониторинг, осадка, превышение, геометрическое нивелирование, тригонометрическое нивелирование, средняя квадратическая погрешность

\section{CREATION OF A HIGH-ALTITUDE GEODESIC CONTROL FOR DEFORMATION MONITORING OF CAPITAL CONSTRUCTION FACILITIES}

\section{Evgeny I. Avrunev}

Siberian State University of Geosystems and Technologies, 10, Plakhotnogo St., Novosibirsk, 630108, Russia, Ph. D., Director of the Institute of Cadastre and Environmental Management, phone: (383)344-31-73, e-mail: kadastr204@yandex.ru

\section{Pavel S. Batin}

LLC «Geocity», 29/1, Titova St., Novosibirsk, 630054, Russia, Director, phone: (903)999-38-26, e-mail: batin866@mail.ru

Nikolai O. Grevtsev

Siberian State University of Geosystems and Technologies, 10, Plakhotnogo St., Novosibirsk, 630108, Russia, Student, phone: (960)797-32-33, e-mail: kadastr204@yandex.ru 
The article defines the relevance of the implementation of trigonometry leveling in geodesic monitoring. At a particular production facility, a comparison of the accuracy of trigonometry leveling performed using two electronic total stations is made with high-precision geometric leveling, which is built according to the program of the first class. Based on the comparison of calculated elevations with reference values derived from high-precision geometric leveling, the real accuracy of trigonometry leveling and the efficiency of the proposed mathematical algorithm to determine tolerances in the use of this most technological way of determining the spatial position of real estate is determined. The proposed mathematical algorithm should be used in geodesic monitoring.

Keywords: capital construction object, deformation monitoring, precipitation, excess, geometric leveling, trigonometry leveling, average square error

\section{Введение}

Одним из актуальных направлений развития земельно-имущественных отношений в Российской Федерации в настоящее время является перевод кадастра недвижимости в формат 3D, а также проведение деформационного мониторинга объектов капитального строительства (ОКС) для определения осадок и деформаций зданий и сооружений [1-5]. Реализация этого направления необходима для создания единого трехмерного геопространства территориального образования, в котором возможно решение многочисленных задач устойчивого развития территорий и реализации принятой и утвержденной концепции «Умный город».

Поэтому, в настоящее время, возникла настоятельная необходимость в определении методов и исследовании точности построения геодезического обоснования, позволяющего определять пространственные характеристики объектов недвижимости. Многими авторами выполнены фундаментальные научные исследования в области определения плоских прямоугольных координат, характеризующих плановое местоположение ОКС, что, к сожалению, нельзя сказать об определении его пространственного положения в высотном отношении [2, 6-7]. Для решения этой задачи наиболее точным и часто применяемым способом построения высотного геодезического обоснования является метод геометрического нивелирования, который имеет весьма серьезные ограничения по диапазону измерения превышений [4] и, следовательно, не является наиболее технологичным при построении высотных геодезических сетей.

\section{Постановка проблемы}

При построении пространственного или высотного геодезического обоснования наиболее приемлемым вариантом с точки зрения технологии и, соответственно, трудоемкости, вне всякого сомнения, является метод тригонометрического нивелирования, выполняемого с использованием современных электронных тахеометров. Однако, в соответствии с научно-техническими публикациями, посвященными данной тематике [4], тригонометрическое нивелирование по точности измерений существенно уступает геометрическому нивелированию. Поэтому, к сожалению, в нормативно-правовой литературе нет доста- 
точного отражения по определению допустимых областей применения этого, несомненного перспективного и наиболее технологического способа для построения высотного геодезического обоснования, необходимого при проведении деформационного мониторинга ОКС. В частности, не определены допуски на расхождения отметок, вычисленных в сериях наблюдений при измерениях вертикальных углов, не установлены допуски на предельные величины невязок при проложении ходов тригонометрического нивелирования [8].

\section{Алгоритм решения проблемы}

Для выполнения научно-технических исследований, посвященных этой актуальной тематике, был выбран производственный объект, расположенный на улице Семьи Шамшиных в городе Новосибирск. На контролируемом здании были заложены деформационные марки, по которым выполнено высокоточное геометрическое нивелирование короткими лучами, соответствующее всем требованиям нормативно-правовых документов [8].

Назначение выполнения производственных работ заключалось в проведении деформационного мониторинга для составления деформационного паспорта объекта и проведения соответствующих мероприятий в случае возникновения недопустимых осадок и деформаций контролируемого ОКС. Расположение контролируемого объекта на топографическом плане и схема построения ходов высокоточного геометрического нивелирования приведена на рисунке.

Ход высокоточного геометрического нивелирования построен в соответствии со всеми требованиями нормативно-правовых документов по программе нивелирования первого класса с оценкой точности выполненных измерений. Заключительным контролем явилось вычисление невязки в замкнутом ходе, которая вычислялась по следующей формуле и которая не превосходила предельно допустимых значений $(\mathrm{f}=+0,3$ мм $)$

$$
f_{\text {доП }}=0,3 \cdot \sqrt{\sum n_{i}}=0,3 \cdot \sqrt{13}=1,1 \text { мм, }
$$

где $\mathrm{n}$ - число секций в нивелирном ходе.

Следовательно, отметки деформационных марок и вычисленные по их значениям превышения могут использоваться как эталонные при анализе точности ходов тригонометрического нивелирования.

Предложенная методика тригонометрического нивелирования заключалась в следующем: для повышения точности и надежности результатов измерение вертикальных углов и длин линий выполнялось двумя электронными тахеометрами Sokkia SET 630RK и Trimle M3DR, инструментальные точности которых составляют $\mathrm{m}_{\beta}=5$ ". Измерение углов выполнялось шестью приемами при двух положениях вертикального круга, длины визирных лучей не превышали 50 м. 


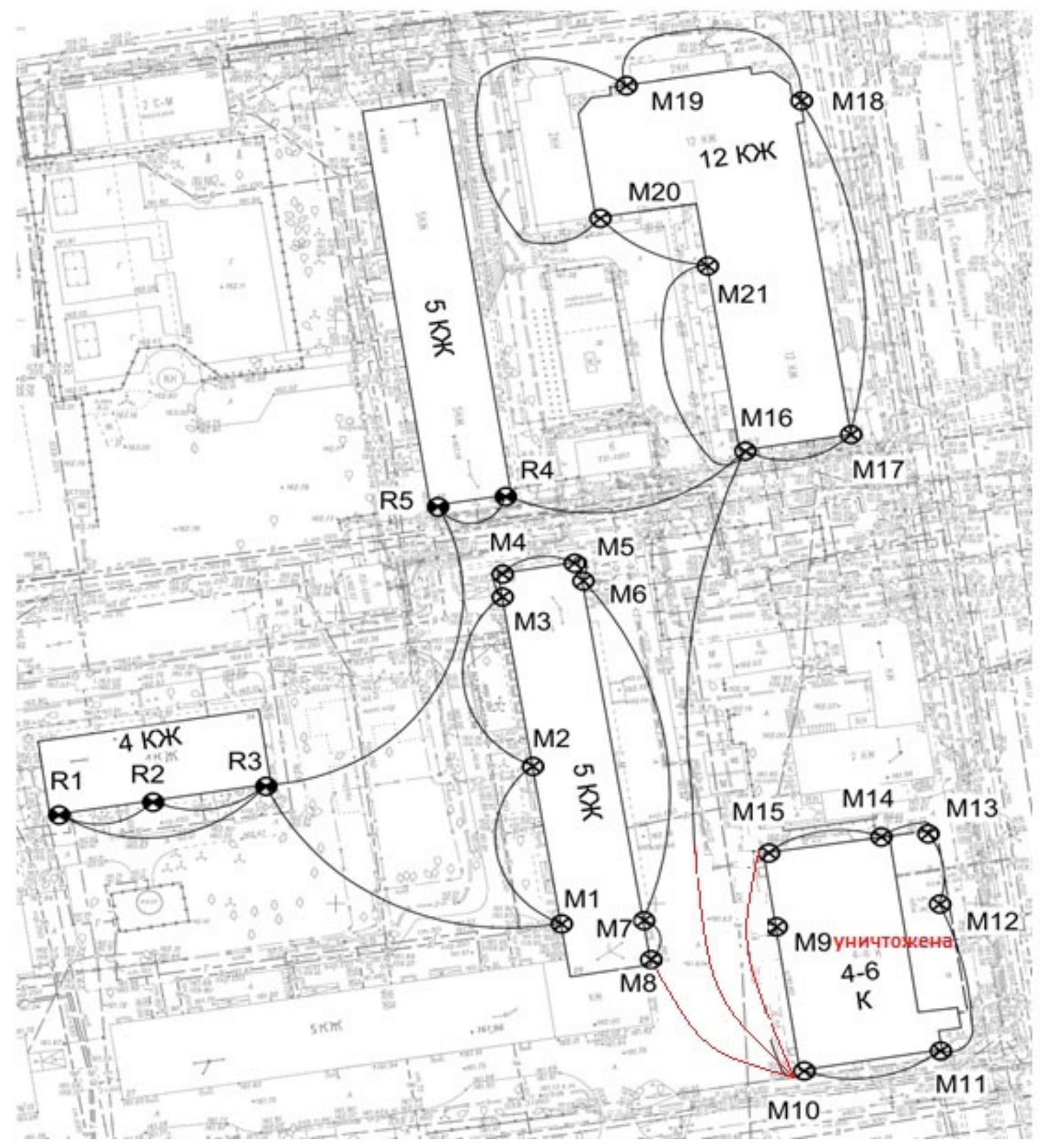

Схема ходов высокоточного геометрического нивелирования короткими лучами

Расхождение отметок внутри серии наблюдений вычислялось на основании следующей формулы, в предположении незначительных значений вертикальных углов (углов наклона)

$$
\Delta_{D}=t \cdot m_{H} ; \quad \frac{m_{H}}{S}=\frac{m_{\gamma}}{\rho^{\prime \prime}} ; \quad m_{H}=\frac{m_{\gamma} \cdot S}{\rho^{\prime \prime}} ; \quad \Delta_{D}=t \cdot \frac{m_{\gamma} \cdot S}{\rho^{\prime \prime}}=2 \cdot \frac{5^{\prime \prime} \cdot 50000 \mathrm{MM}}{206265^{\prime \prime}}=2,4 \mathrm{MM} .
$$

где $\mathrm{m}_{\gamma}$ - инструментальная точность измерения вертикального угла электронным тахеометром; 
$\mathrm{S}$ - максимальная длина визирного луча от электронного тахеометра до деформационной марки.

При определении отметок деформационных марок двумя электронными тахеометрами, которые характеризуются одинаковой инструментальной точностью, СКО и допуск на расхождение отметок предлагается вычислять по следующей формуле

$$
\Delta_{D}=t \cdot m_{H} ; \quad m_{H}=\frac{m_{\gamma} \cdot S}{\sqrt{2} \cdot \rho^{\prime \prime}} ; \quad \Delta_{D}=t \cdot \frac{m_{\gamma} \cdot S}{\rho^{\prime \prime}}=2 \cdot \frac{5 " \cdot 50000 \mathrm{MM}}{\sqrt{2} \cdot 206265^{\prime \prime}}=1,7 \mathcal{M M}, \quad m_{H}=0,8 \mathrm{MM} .
$$

При принятии гипотезы о независимости вычисленных отметок между собой, СКО превышения, представленная как функция отметок деформационных марок, определится по следующей формуле

$$
m_{h}=\sqrt{m_{H_{1}}^{2}+m_{H_{2}}^{2}}=\sqrt{2} \cdot m_{H}=\sqrt{2} \cdot \frac{m_{\gamma} \cdot S}{\sqrt{2} \cdot \rho^{\prime \prime}}=\frac{m_{\gamma} \cdot S}{\rho^{\prime \prime}}=\frac{5^{\prime \prime} \cdot 50000 \mu M}{206265^{\prime \prime}}=1.2 \mathrm{MM} .
$$

Следовательно, допустимая невязка в замкнутом ходе тригонометрического нивелирования при использовании электронных тахеометров с соответствующей инструментальной точностью $\mathrm{m}_{\gamma}$, при максимальных длинах визирных лучей до определяемых деформационных марок $\mathrm{S}=50$ м. вычисляется по следующей формуле

$$
\Delta_{D}=t \cdot m_{h} \cdot \sqrt{n}=t \cdot \frac{m_{\gamma} \cdot S}{\rho^{\prime \prime}} \cdot \sqrt{n}=2 \cdot \frac{5^{\prime \prime} \cdot 50000 \mu u}{206265^{\prime \prime}} \cdot \sqrt{10}=7,6 \mu \mu .
$$

\section{Результаты}

Выполненные вычисления по уравниванию хода тригонометрического нивелирования полностью подтвердили сделанные теоретические выкладки по определению предельно допустимых точностных параметров. Все вычисленные параметры построенного хода соответствуют установленным нормативным допускам.

Сравнение уравненных превышений, полученных в результате выполнения геометрического и тригонометрического нивелирования, приведены в таблице.

В таблице приняты следующие обозначения:

$\mathrm{H}_{1}, \mathrm{H}_{2}$ - отметки деформационных марок, полученные в результате тригонометрического нивелирования независимо двумя тахеометрами в результате измерений шестью приемами;

$\Delta_{1}$ - расхождение между результатами наблюдений двух тахеометров;

$\Delta_{2}-$ расхождение между геометрическим и тригонометрическим нивелированием. 
Анализ точности превышений из тригонометрического нивелирования

\begin{tabular}{|c|c|c|c|c|c|c|}
\hline № & $\mathrm{h}_{\text {ГЕОМ }}$ & $\mathrm{H}_{1}$ & $\mathrm{H}_{2}$ & $\mathrm{~m}_{1}$ & $\mathrm{~h}_{\text {тРИГ }}$ & $\mathrm{m}_{2}$ \\
\hline 4 & & 100,939 & 100,94 & $-0,001$ & & \\
\hline & $-0,0815$ & & & & $-0,0785$ & $-0,003$ \\
\hline M5 & & 100,861 & 100,861 & 0 & & \\
\hline & 0,1891 & & & & 0,1855 & 0,004 \\
\hline M6 & & 101,046 & 101,047 & $-0,001$ & & \\
\hline & & & & & & \\
\hline M10 & & 100,161 & 100,162 & $-0,001$ & & \\
\hline & $-0,0578$ & & & & $-0,0580$ & 0,000 \\
\hline M11 & & 100,103 & 100,104 & $-0,001$ & & \\
\hline & 0,0589 & & & & & \\
\hline M12 & & & & & & \\
\hline & 0,0717 & & & & & \\
\hline M13 & & 100,228 & 100,228 & 0 & & \\
\hline & $-0,0752$ & & & & $-0,0715$ & $-0,004$ \\
\hline M14 & & 100,156 & 100,157 & $-0,001$ & & \\
\hline & 0,3402 & & & & 0,3420 & $-0,002$ \\
\hline M15 & & 100,498 & 100,499 & $-0,001$ & & \\
\hline
\end{tabular}

Анализ точности превышений из тригонометрического нивелирования выполнялся с использованием формулы Гаусса

$$
m=\sqrt{\frac{\sum_{i=1}^{n} \Delta_{l}^{2}}{n}},
$$

где $\Delta_{\mathrm{i}}$ - расхождение между значениями из наблюдений, выполненными различными тахеометрами, или в результате сравнения тригонометрического и геометрического нивелирования;

$\mathrm{n}$ - число анализируемых превышений.

В результате анализа полученных результатов следует отметить, что расхождение между наблюдениями двумя тахеометрами, вычисленное по формуле (6), составляет $\Delta_{1}=0,002$ м, и соответственно СКО определения превышений из геометрического и тригонометрического нивелирования соответственно $\Delta_{2}=$ $0,003 \mathrm{M}$. 


\section{Заключение}

Следовательно, исходя из требований нормативно-правовых документов для определения деформаций данного объекта недвижимости с нормативной точностью $\mathrm{m}=1$ мм, данный вид геодезических измерений использовать не рекомендуется.

Для повышения точности измерений необходимо использовать или более точные электронные тахеометры, например, с СКО измерения вертикальных углов $\mathrm{m}_{\gamma}=1$ " или 2", или уменьшать длины линии визирования, например, до 25 метров, но в этом случае теряется один из главных преимуществ этого способа нивелирования. Следует также ограничивать число определяемых превышений в ходе тригонометрического нивелирования, например, не более 10.

Следует отметить, что СКП измерений, выполняемых при геодезическом мониторинге зданий и сооружений $\mathrm{m}_{\mathrm{H}}$, зависит от их конструкции и типов грунтов, на которых они расположены [8]. Необходимая точность при этом вычисляется по следующей формуле

$$
m_{H O P M}=0,2 \cdot \Delta,
$$

где $\Delta$ - предельно допустимое значение осадки, определенное соответствующим нормативно-правовым документом [8].

Следовательно, необходимую инструментальную точность используемых в тригонометрическом нивелировании электронных тахеометров, рекомендуется определять на основании предложенного математического алгоритма.

\section{БИБЛИОГРАФИЧЕСКИЙ СПИСОК}

1. Карпик А. П. Анализ состояния и проблемы геоинформационного обеспечения территорий // Изв. вузов. Геодезия и аэрофотосъемка. - 2014. - № 4. - С. 3-7.

2. Аврунев Е.И. Технологические аспекты построения 3D-модели инженерных сооружений в городах арктического региона РФ [Текст]/, А. В. Чернов, А. В. Дубровский, А. В. Комиссаров, Е. Ю. Пасечник // Известия Томского политехн. ун-та. Инжиниринг георесурсов. - 2018. - Т. 329, № 7. - С.131-137.

3. Николаев Н.А., Чернов А. В. Трехмерный кадастр недвижимости как новая ступень развития кадастровых систем // Интерэкспо ГЕО-Сибирь-2014 : Х Междунар. науч. конгр., 8-18 апр. 2014 г., Новосибирск; Междунар. науч. конф. "Экон. развитие Сибири и Дальнего Востока. Экономика природопользования, землеустройство, лесоустройство, управление недвижимостью": сб. материалов в 2 т. - Новосибирск : СГГА, 2014. - Т. 2. - С. 194-198.

4. Никонов А.В. Методика тригонометрического нивелирования первого и второго разрядов // Изв. вузов. Геодезия и аэрофотосъемка. - 2015. - №5/С. - С. 39-45.

5. Чернов А.В. Исследование вариантов построения 3D-модели объектов недвижимости для целей кадастра // Вестник СГУГиТ. - 2018 - Т. 23 (3). - С. 192-210.

6. Аврунев Е.И., Колмогоров В.Г., Новоселов Ю.А., Труханов А.Э. Инвентаризация сведений ГКН о местоположении границ земельных участков и объектов капитального строительства. // Геодезия и аэрофотосъемка Известия высших учебных заведений. - 2014. №4/C - C. 181-184. 
7. Карпик А.П., Варламов А.А., Аврунев Е.И. “Совершенствование методики контроля качества спутникового позиционирования при создании геоинформационного пространства территориального образования" Геодезия и аэрофотосъемка Известия высших учебных заведений №4/с (ВАК), 2014г. 185- 188c.

8. СП 126.13330.2017 Геодезические работы в строительстве. СНиП 3.01.03-84 http://docs.cntd.ru/document/550965720

(С) Е. И. Аврунев, П. С. Батин, Н. О. Гревцев, 2021 